\title{
Isolated perforation of the gall bladder following blunt abdominal trauma
}

\author{
D. A. LAFFeY \\ B.Sc., M.B., Ch.B. \\ D. J. HAY \\ M.B., F.R.C.S. \\ Department of Surgery, University of Manchester Medical School, Hope Hospital, Salford M6 8HD
}

\begin{abstract}
Summary
Isolated perforation of the gall bladder as a consequence of blunt abdominal injury is rare. A single case is described which illustrates several features which may characterize this lesion.
\end{abstract}

\section{Introduction}

Rupture of the gall bladder is a well recognized complication of acute cholecystitis. The gall bladder may be damaged along with other viscera in cases of massive abdominal injury or when penetrating wounds occur. Isolated perforation of the gall bladder as a result of non-penetrating injury to the abdomen is a very much rarer occurrence. In his review of the literature, Gayen (1973) found 51 previously reported cases. An apparent increase in the incidence of this lesion has been ascribed by several authors (Schecter, 1969; Fletcher, 1972) to a rise in the numbers of automobile accidents with rapid deceleration and impact against a steering wheel or seat belt as the probable mechanism of injury.

\section{Case report}

A 51-year-old man was admitted to hospital as an emergency. An itinerant with a long history of heavy alcohol abuse, he was a resident in a reception centre. Two and a half weeks before admission, whilst under the influence of alcohol, he had been attacked and kicked in the abdomen. Shortly after the incident he was seen by a doctor who noted some tenderness and guarding in the right upper quadrant and as the patient was otherwise well, a presumptive diagnosis of superficial injury was made and no specific treatment was offered.

In the period up to his admission, the patient became anorexic and began to vomit all solid food and for the week before his admission to hospital, his intake had been restricted to fluids. In addition, his abdomen had become more painful and had swollen progressively. During this period, his bowels had continued to function at least once daily, with the production of watery green or yellow stools. He also suffered sweating attacks.

On examination he was cachectic, afebrile, not shocked or anaemic and with no jaundice or stigmata of liver disease. His abdomen was distended with fluid and was generally tender with some guarding. Bowel sounds were present although reduced and rectal examination revealed yellow, foul-smelling stools. Investigations on admission showed: haemoglobin $14.4 \mathrm{~g} / \mathrm{dl}$., PCV 0.402, WBC $11.5 \times 10^{9} / 1$, with a normal differential. Urea and electrolytes were normal and the serum amylase was 266 Somogyi units $/ 100 \mathrm{ml}$. The chest X-ray was clear and the abdominal X-rays served only to confirm the presence of ascites. Paracentesis abdominis was performed and more than 3 litres of dark yellow fluid were removed. Following this procedure, the patient's symptoms were much relieved and enabled a more thorough clinical examination of the abe domen, which was found to be minimally tendef with no rigidity. No masses were detectable an $\Phi$ bowel sounds were normal. It was decided to treat this patient conservatively, at least until the results of the analyses of the ascitic fluids were known. He was at first able to tolerate a light diet, but over the course of the next 4 days, with the rapid reaccumulation of ascites came a return of the vomiting and abdominal pain together with the appearance of jaundice. Liver function tests during this period showed total bilirubin $52 \mathrm{mmol} / 1$ (3-17), alkaline phosphatase $640 \mathrm{u} . / 1(20-95)$ and albumin $31 \mathrm{~g} / 1$ (36-52).

In view of the rapid deterioration of the patient's condition, it was decided to perform a laparoscopy which showed gross bile staining of the peritoneal cavity. Laparotomy was performed immediately and this showed gross biliary contamination of the whole peritoneal cavity with multiple collections of fibrinous exudate between loops of bowel, in the pelvis, in both paracolic gutters and beneath both hemi-diaphragms. The thin walled, otherwise normal gall bladder was perforated by a small tear in its fundus. There were no gall stones. The pancreas was very thickened and there was marked fat necrosis. A pre-operative cholangiogram showed no other abnormality in the biliary tree and cholecystectomy was performed, together with drainage of the common bile duct with a T-tube. Drains were 
placed in each paracolic gutter and beneath the left hemidiaphragm and brought out through separate stab wounds.

The patient was placed on gentamicin and lincomycin.

The postoperative course was uneventful. A Ttube cholangiogram performed 8 days postoperatively, showed a normal picture and the patient was discharged 2 weeks after operation.

Samples of ascitic fluid taken at the time of paracentesis abdominis were sent for microbiological examination and all attempts at culture were negative and no evidence of bacterial contamination was seen on microscopic examination. Cultures for acid-fast bacilli were also negative.

\section{Discussion}

A review of previous publications shows that isolated traumatic perforation of the gall bladder occurs predominantly in males, in keeping with their propensity for involvement in violent incidents. Several distinct groups of patients can be defined. Children, especially those involved in road accidents, form one such group (Schecter, 1969) their susceptibility presumably relating to their increased vulnerability to direct abdominal trauma and possibly also to their less well developed anatomical protection of the gall bladder.

Another important group consists of older males who, whilst under the influence of alcohol, sustain abdominal injuries, either as a result of assault (Solheim, 1972; Fielding and Strachan, 1975; Brickley et al., 1960), or as a result of steering wheel or seat belt trauma in road accidents (Breen, 1975; Wright, 1976).

For a healthy gall bladder to rupture, distension at the time of trauma must be considered almost an essential prerequisite. This may occur in obstructive biliary tract disease, after a meal (Knepper, Riddell and McDavid, 1956), or after alcohol. Alcohol appears to cause an increase in pressure in the biliary tree (Pirola and Davis, 1968), an effect probably mediated by the gastrin- and secretin-releasing effect of alcohol in the stomach and duodenum (Wright, 1976). Brickley et al. (1960) collected a series of 3 cases which occurred within months of each other, all in middle-aged alcoholic men who had been drinking before the time of injury.

The vast majority of reported cases have occurred in patients with otherwise disease-free biliary tracts. This is related to the predominant distribution of cases in the lower age groups. It may also be argued that a chronically inflamed sclerotic gall bladder may offer more resistance to trauma than its thin walled, healthy counterpart.

The clinical picture which is seen in most of the reported cases of traumatic isolated perforation of the gall bladder has been well summarized in previous reviews (Estrada and Sutherland, 1969; Schecter, 1969). Characteristically, following the initial injury, symptoms are few, usually relatively mild and there is little constitutional upset. The initial symptoms often regress and there may follow what Schecter has termed a 'period of illusion' in which the patient's condition may improve to an extent to lead to discharge from hospital (Estrada and Sutherland, 1969; Breen, 1975). This period may last for a matter of hours, several days or even weeks and gradually gives way to increasing abdominal discomfort and collection of ascites.

There have been several reports of delayed perforation of the gall bladder following blunt trauma (Fielding and Strachan, 1975; Brickley et al., 1960), in which the clinical course and operative findings have been such as to suggest that gross biliary contamination of the peritoneal cavity occurred some considerable time after the initial trauma. This has been explained in terms of late perforation of a contused area of gall bladder or of loss of omental sealing as a late event.

Delay in making the diagnosis is the rule rather than the exception. Many cases have been reported where the delay is a matter of weeks rather than hours (Fletcher, 1972). Diagnosis is rarely made before operation.

Non-traumatic perforation of the gall bladder as may occur in acute cholecystitis is much commoner than traumatic perforation. Spillage of infected bile gives rise to the picture of 'classical' biliary peritonitis with acute onset of toxaemia and shock, and associated very high mortality variously estimated at between $30 \%$ and $90 \%$ (Ellis and Adair, 1974; MacDonald, 1966). In the great majority of recorded cases of traumatic perforation, however, there has been no pre-existing biliary tract disease. Leakage of sterile bile gives rise to a clinical picture, exemplified by the case described, which bears little resemblance either in symptomatology or in prognosis to the more common 'classical' biliary peritonitis. The overall mortality in traumatic perforation of the gall bladder has been estimated at about 5\% (Schecter, 1969). Breen (1975) in his search of the literature was unable to find recorded a single death attributable to traumatic rupture of the gall bladder as an isolated injury.

Biochemical tests are of very limited value in making an early diagnosis. Liver function tests may be of value in revealing diversion of the bile stream. Peritoneal lavage offers the greatest prospect of reaching the diagnosis with the provisos that falsepositive results may be obtained in cases of ruptured foregut or even in acute pancreatitis and false negative results are possible if bile leakage is entirely retroperitoneal. 
In several important respects the case presented accords with previous examples of this rare condition, namely the association with alcohol ingestion, the protracted course with delay in diagnosis and the comparatively benign nature of this form of biliary peritonitis. It is suggested that a wider appreciation of the essential differences between the clinical picture resulting from contamination of the peritoneal cavity with sterile bile as compared with infected bile might lead to earlier diagnosis in these cases.

\section{Acknowledgment}

We wish to thank Mr G. Ingram, under whose care this patient was, and Mrs E. W. Robinson, for typing the manuscript.

\section{References}

BREeN, P.C. (1975) Rupture of the gall bladder after blunt abdominal trauma. Southern Medical Journal, 68 (5), 658.

Brickley, H.D., Kaplan, A., Freeark, R.J. \& Broccolo, E. (1960) Immediate and delayed rupture of the extrahepatic biliary tract following blunt abdominal trauma. American Journal of Surgery, 100, 107.
Ellis, H. \& AdaIR, H.M. (1974) Bile peritonitis - a report of fifteen patients. Postgraduate Medical Journal, 50, 713.

Estrada, R.L. \& Sutherland, N.G. (1969) Subparietal or non-penetrating traumatic rupture of the biliary tree. British Journal of Surgery, 56, 85.

Fielding, J.W.L. \& Strachan, C.J.L. (1975) Jaundice as a sign of delayed gall bladder perforation following blunt abdominal trauma. Injury, 7, 66.

FLETCHER, W.S. (1972) Non-penetrating trauma to the gall bladder and extrahepatic bile ducts. Surgical Clinics of North America, 52, 711.

GAYEN, S. (1973) Rupture of the gall bladder due to nonpenetrating injury to the abdomen. Journal of the Royal College of Surgeons of Edinburgh, 18, 242.

KNEPPER, P.A., RidDell, R.V. \& MCDAVID, J.R. (1956) Traumatic rupture of the gall bladder without a penetrating wound of the abdominal wall. Archives of Surgery, 73, 371.

MACDONALD, J.A. (1966) Perforation of the gall bladder associated with acute cholecystitis. Annals of Surgery, 164, 849.

Pirola, R.C. \& Davis, A.E. (1968) Effects of alcohol on sphincteric resistance at the choledochoduodenal junction in man. Gut, 9, 557.

SCHECTER, D.C. (1969) Solitary wounding of the gall bladder from blunt abdominal trauma. New York State Medical Journal, 69, 2895.

Solheim, K. (1972) Blunt gall bladder injury. Injury, 3, 246.

Wright, J.E. (1976) Gall bladder rupture, an isolated seat belt injury. Medical Journal of Australia, 21, 785. 